Gunnar Flötteröd, Kai Nagel

\title{
High speed combined micro/macro simulation of traffic flow
}

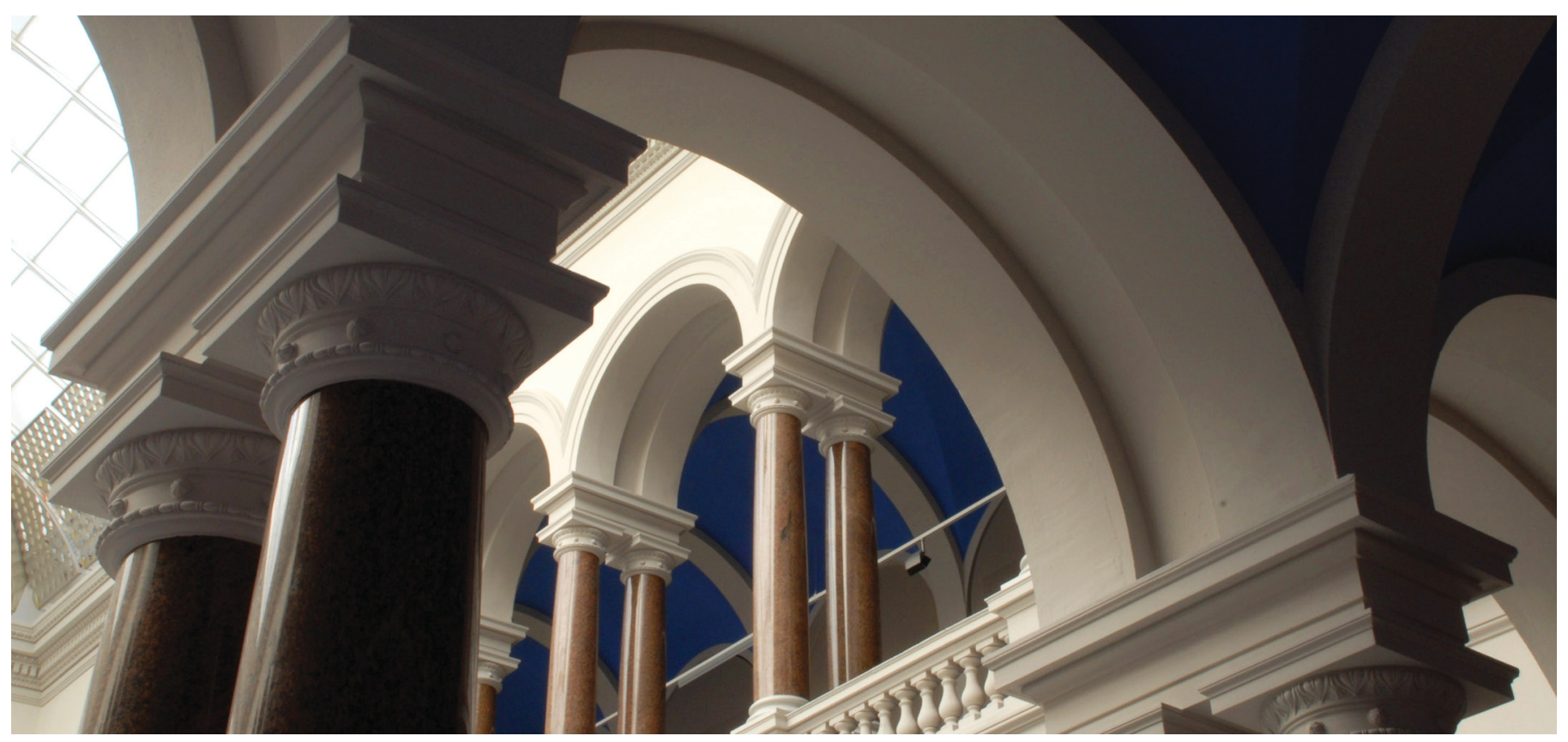

Flötteröd, G.; Nagel, K. (2007). High Speed Combined Micro/Macro Simulation of Traffic Flow. 2007 IEEE

Intelligent Transportation Systems Conference. https://doi.org/10.1109/ITSC.2007.4357782 


\title{
High Speed Combined Micro/Macro Simulation of Traffic Flow
}

\author{
Gunnar Flötteröd* and Kai Nagel**
}

\begin{abstract}
We describe two new and practically relevant simulation techniques related to the Kinematic Wave Model of traffic flow. Firstly, we demonstrate how the well-known Godunov solution scheme can be run on variable time scales in a computationally very efficient way. Secondly, we demonstrate how the resulting macroscopic traffic flow model can be run in conjunction with a microscopic model of driver behavior while maintaining high computational performance.
\end{abstract}

\section{INTRODUCTION}

This article describes two features of a newly developed high performance mixed micro/macro mobility simulation based on the Kinematic Wave Model (KWM) [1], [2].

Firstly, in Section II the well-known Godunov solution approach to the KWM is applied in conjunction with a simulation logic that runs different model components on variable time scales. Simulation performance is considerably improved by this feature.

Secondly, it is shown in Section III how this macroscopic model can be run in combination with a microscopic representation of individual travelers. This coupling (i) preserves the analytical features of the macroscopic model, (ii) significantly reduces vehicle discretization noise, and (iii) maintains the high computational speed of the aforementioned simulation scheme on variable time scales.

Due to the twofold purpose of this article, further introductory notes are given in the according sections of the text.

\section{Running the Kinematic Wave Model ON VARIABle TIME SCALES}

The arguably best known approaches for numerical simulation of the KWM are the Cell-Transmission Model (CTM) [3], [4] and the STRADA model [5], [6]. Both are based on the Godunov method, e.g. [7]. Due to space restrictions the workings of these models can only conceptually be recapitulated. Reference [8] is recommended as a more comprehensive introduction.

The possibility to improve the computational speed of the CTM by variation of its spatio temporal discretization has already been recognized shortly after the model's appearance [9]. This problem is reconsidered in this work and enhanced towards combined micro/macro simulation.

\footnotetext{
* Gunnar Flötteröd is with the Group for Transport Systems Planning and Transport Telematics, Technische Universität Berlin, D-10587 Berlin, Germany. Tel: +49-30-314-29520, fax: +49-30-314-26269, mail: floetteroed@vsp.tu-berlin.de.

**Kai Nagel is head of the Group for Transport Systems Planning and Transport Telematics, mail: nagel@vsp.tu-berlin.de.
}

\section{A. Simulation Logic}

Discrete time simulation is straightforward if a uniform time step length $T$ is used. Every link with maximum velocity $\hat{v}$ is disassembled into cells of

$$
\text { minimum discretization length }=T \hat{v} .
$$

The amount of macroscopic vehicles stored in cell $i$ at discrete simulation time step $k$ is denoted by $x_{i}(k)$.

Demand function $\Delta_{i}\left(x_{i}(k)\right)$ and and supply function $\Sigma_{i}\left(x_{i}(k)\right)$ specify the maximum rate (in vehicle units per second) at which vehicles can leave and enter cell $i$ during time step $k$ depending on this cell's current occupancy $x_{i}(k)$. These functions constitute a generalization of the fundamental diagram [10] where the demand function corresponds to the fundamental diagram's uncongested half and the supply function to its congested complement [8]. Demand and supply constitute the boundaries of a cell.

Calculation of actually transmitted flows is the task of network elements we denote as connectors. One such connector is placed between every set of adjacent cells. Phenomenological constraints can be incorporated in its workings. Of major importance to this work are flow splitting fractions at diverges. Parameter $\beta_{i j}(k)$ specifies which proportion of the flow leaving upstream cell $i$ at time step $k$ enters downstream cell $j$. The actually transmitted outflow (inflow) rate of a cell $i$ as calculated by its downstream (upstream) connector at time step $k$ is denoted by $q_{i}^{\text {out }}(k) \in\left[0, \Delta_{i}\left(x_{i}(k)\right)\right]\left(q_{i}^{i n}(k) \in\right.$ $\left.\left[0, \Sigma_{i}\left(x_{i}(k)\right)\right]\right)$. Various specifications of this general flow transmission formalism are possible [4], [11], [8].

Conceptually, a simulation step (tick) consists of two parts:

1) Every connector calculates vehicle transmissions between its adjacent cells according to their boundaries.

2) Every cell updates its occupancy according to these transmissions, which specifies its boundaries for the next tick.

Simulation of a heterogeneous urban network requires relatively small cells to model densely meshed regions. This calls for a small $T$ and in turn implies an unnecessarily precise modeling of longer road segments. The use of larger cells running on the same temporal grid somewhat mildens this problem. However, a significant share of urban network computations is incurred by the intersection logic. Thus, a simulation scheme that minimizes the number of simulation ticks themselves is needed.

Spatio-temporal dynamics within an isolated link are uniquely defined if an initial density profile as well as 
feasible upstream inflows and downstream outflows are provided. Given an individually chosen time step length and an appropriate spatial discretization, standard CTM logic facilitates a KWM-consistent numerical simulation. Since all spatial dynamics are enclosed within the link, it can be viewed from the outside as a discrete-time nonlinear ordinary dynamical system with two inputs (in- and outflows) and two outputs (its upstream and downstream boundaries). The same argument holds for individual cells. Likewise, any pointlike intersection model represented by a connector that is consistent with the KWM constitutes for any chosen time step length a memoryless nonlinear discrete-time system with upstream flow demands and downstream flow supplies as inputs and resulting vehicle transmissions as outputs.

Adopting a technical point of view, these systems can immediately be joined: Outputs of systems with a large time step are held constant when needed as inputs for faster ticking systems, and outputs of faster ticking systems are averaged before they are fed into slower ticking systems. Since such holding and averaging affect system dynamics mainly in terms of a delay that is proportional to the involved time step length, a reasonable balance between additionally introduced imprecision and computational speedup can be achieved. This is confirmed by the experimental results given in Section II-A.4.

This simulation scheme is detailed in the following. A cell $i$ (connector $c$ ) is denoted as "due" at discrete simulation time step $k$ if $k$ is an integer multiple of its individual time step length $T_{i}\left(T_{c}\right)$. A simulation time step's duration is generally assumed to be 1 second. Two procedures are executed at every $k$ :

1) Every cell $i$ that is due according to its individual time step length $T_{i}$ calculates supply and demand boundaries from its current occupancy and keeps these results constant for the next $T_{i}$ seconds.

2) Every connector $c$ that is due according to its individual time step length $T_{c}$ calculates average flow rates that hold for the next $T_{c}$ seconds and notifies its adjacent cells of the resulting vehicle transmissions.

Sections II-A.1, II-A.2, and II-A.3 specify the technicalities of this simulation scheme.

1) Cell Boundaries: Every cell $i$ has exactly one preceding one succeeding connector. While $i$ has an individual time step length $T_{i}$, it is embedded in a system potentially running at a 1 -second time scale. This requires its supply $\Sigma_{i}\left(x_{i}(k)\right)$ and demand $\Delta_{i}\left(x_{i}(k)\right)$ to be defined at every second. Since these boundaries are static functions only of $i$ 's occupancy, it is sufficient to specify $x_{i}$ for every simulation time step by

$$
x_{i}\left(m T_{i}+n\right)=x_{i}\left(m T_{i}\right) \quad m \in \mathbb{N}, n \in\left\{0, \ldots, T_{i}-1\right\} .
$$

2) Connector Flow Rate Update: Consider a connector $c$ with a set $\mathcal{P}_{c}$ of preceding and a set $\mathcal{S}_{c}$ of succeeding cells. Its time step length $T_{c}$ is chosen such that $(i)$ the connector recalculates flow rates whenever an adjacent cell boundary changes and (ii) the overall computational load is minimized. This is achieved by choosing $T_{c}$ as the largest common divisor (lcd) of all adjacent cell time step durations

$$
T_{c}=\operatorname{lcd}_{i \in \mathcal{P}_{c} \cup \mathcal{S}_{c}}\left(T_{i}\right) .
$$

Arbitrary cell time step durations might yield low computational savings because of possibly small $T_{c}$ resulting from this equation, so they are constrained to be powers of two. This turns the connector time step length into the minimum of its adjacent cell time step durations.

3) Cell State Update: Even if a cell $i$ 's state $x_{i}$ changes only every $T_{i}$ seconds, its adjacent connectors might run at a higher frequency. On the finest temporal scale this implies

$$
\begin{aligned}
x_{i}\left(m T_{i}+T_{i}\right) & =x_{i}\left(m T_{i}\right) \\
& +1 \mathrm{~s} \sum_{n=0}^{T_{i}-1}\left(q_{i}^{\text {in }}\left(m T_{i}+n\right)-q_{i}^{\text {out }}\left(m T_{i}+n\right)\right) .
\end{aligned}
$$

Denote by $p_{i}\left(s_{i}\right)$ the preceding (succeeding) connector of cell $i$. Because of Eq. (3), $T_{i} / T_{p_{i}}$ and $T_{i} / T_{s_{i}}$ are integer values. This allows for the following simplification:

$$
\begin{aligned}
x_{i}\left(m T_{i}+T_{i}\right)= & x_{i}\left(m T_{i}\right)+T_{p_{i}} \sum_{n=0}^{T_{i} / T_{p_{i}}-1} q_{i}^{\text {in }}\left(m T_{i}+n T_{p_{i}}\right) \\
& -T_{s_{i}} \sum_{n=0}^{T_{i} / T_{s_{i}}-1} q_{i}^{\text {out }}\left(m T_{i}+n T_{s_{i}}\right) .
\end{aligned}
$$

Therefore, it is sufficient to notify cell $i$ every $\operatorname{lcd}\left(T_{p_{i}}, T_{s_{i}}\right)$ seconds of possible flow rate changes. This is done independently by its upstream and downstream connector every $T_{p_{i}}$ and $T_{s_{i}}$ seconds by transmission of the appropriate addend in Eq. (5) to the cell. Since the cell's boundaries are held constant for a possible longer duration according to Eq. (2), transmitted vehicles are intermediately cached by the cell.

4) Experimental Investigation of Simulation Precision: A linear test network has been set up. It consists of a sequence of 5 identical links with a space capacity of $140 \mathrm{veh} / \mathrm{km}$, a flow capacity of $2000 \mathrm{veh} / \mathrm{h}$, a maximum velocity of $50 \mathrm{~km} / \mathrm{h}$, and a length of $32 \cdot \hat{v} \cdot 1 \mathrm{~s} \approx 444.44 \mathrm{~m}$ each. The initial simulation boundaries resemble the original CTM validation setting [3]: A linear density gradient from zero to maximum density is placed on the network, with zero density at its upstream and maximum density at its downstream end. No traffic is allowed to enter or leave the network. The simulation is run until a steady state is reached.

Figure 1 shows the resulting space-time plots in various discretization settings. Plot (a) provides a good approximation to the exact solution. Initially, two shockwaves occur: an upstream shockwave moving at positive velocity and a downstream shockwave moving at negative velocity. They merge in the center of the network and persist as a stationary density discontinuity with all traffic being queued up in the downstream half of the network. For comparison, results with a much coarser but still homogeneous discretization are shown in plot (b). 
Fig. 1. Space Time Plots With Variable Spatio Temporal Discretizations

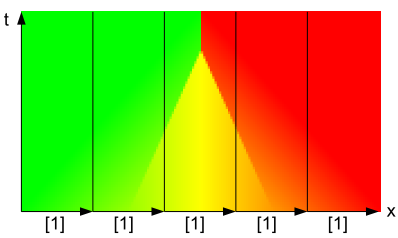

(a) All links have a time step duration of of 1 second and consist of 32 cells.

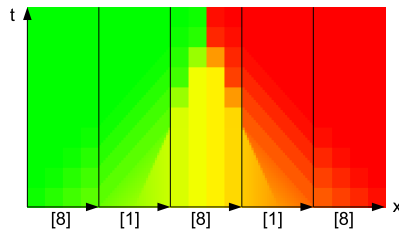

(c) All but the second and fourth link have an 8 second time step.

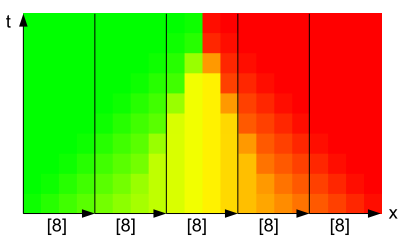

(b) All links have a time step duration of 8 seconds and consist of 4 cells.

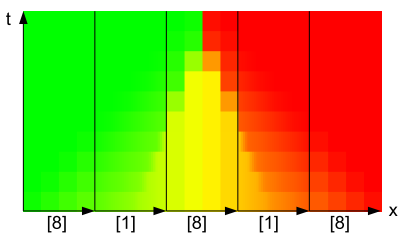

(d) The same data as (c) but averaged on a temporal grid of 8 seconds.

Colors encode densities as follows: green is zero density, yellow is half of maximum density, and red is maximum density. The parenthesized numbers below the links indicate their individual time step duration.

The results with heterogeneous simulation time steps nicely reflect the workings of the underlying Godunov method: At every simulation time step, the Godunov scheme solves a Riemann problem at all cell boundaries. Placing fast ticking cells next to slower cells explicitly displays the resulting shockwaves as it can be seen in plot (c). While these artifacts are unequivocally owed to the simulation scheme on variable time scales, they are put into relation by plot (d). It shows the same result after it has been averaged on a temporal grid according to the largest time step duration originally used. The artefact's are nicely smeared out while the original shockwaves are maintained with precision at least comparable to plot (b).

These results indicate that the overall simulation error remains in the order of the largest involved time step duration, as it has been previously hypothesized. Artifacts can occur at boundaries between slow and fast ticking cells but can also be removed by temporal averaging of simulation output before further processing. No amplification of artifacts is observed.

\section{B. Network Discretization}

Our testcase is modeled after the road network of Greater Berlin, see Figure 2. This network consists of 1083 nodes and 2459 unidirectional links. It is quite heterogeneous: the innerurban area is modeled in relatively high resolution while the surrounding freeway ring is comprised of several links that are many kilometers long.

Network time constant $\hat{T}$ denotes the largest allowed simulation time step duration in the network. It compromises

Fig. 2. Major Road Network of Greater Berlin

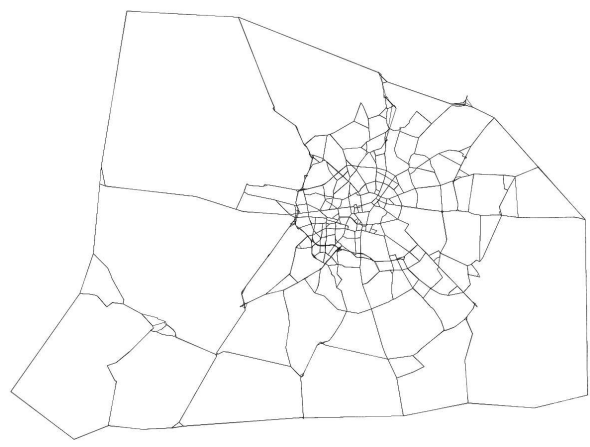

Fig. 3. Effect of Network Time Constant

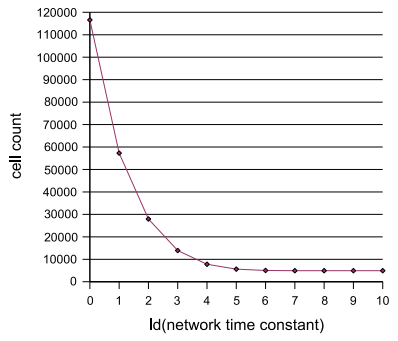

(a) Number of cells over $\log _{2}(\hat{T})$. Network geometry has a limiting effect on cell sizes such that $\hat{T}$ value beyond $2^{6} \mathrm{~s}$ do not result in an increased coarsening.

between a high simulation resolution (small $\hat{T}$ ) and high computational performance (large $\hat{T}$ ). The network is discretized such that link-specific simulation time step durations are the largest powers of two not greater than $\hat{T}$ for which Eq. (1) still holds. Additionally, it is required that every link consists of at least two cells.

Figure 3 (a) shows the effect of $\hat{T}$ on the number of cells in the network. With increasing $\hat{T}$ the number of cells approaches its minimum value of $2 \times 2459$. A histogram of intersection connector time step lengths for $\hat{T}=64 \mathrm{~s}$ is given in Figure 3 (b). The high number of intersections with a relatively low time step duration is due to the finely meshed interurban network which is precluded from a "slower" simulation clock.

\section{A MIXED MICRO/MACRO MOBILITY SIMULATION}

Two fundamentally different concepts can be encountered in the literature on coupled micro-macro simulations.

"Hybrid" approaches link simulations that work on different degrees of aggregation at well defined locations in the network, e.g. [12], [13]. This approach is attractive if the required simulation fidelity varies spatially.

"Mesoscopic" simulations move individual vehicles based on aggregated laws of motion in order to increase computational performance while retaining a microscopic representation 
of behavior [14], [15]. Simulation based dynamic traffic assignment usually employs such models, e.g. [16], [17], [18], [19].

The approach described here is a mesoscopic model with a distinct macroscopic aspect.

\section{A. Particle Movement}

1) Specification: Consider a set $\mathcal{M}$ of particles (a "population" of travelers, agents, or vehicles) floating through the network. Particles have no "mass" insofar as they do not contribute to the macroscopic occupancy on a cell. It is assumed that at the time of a particle's entrance into the network, an appropriate amount of macroscopic flow has also been dismissed into the system, resulting in a mass balance between particles and total macroscopic occupancy.

The macroscopic traffic flow model is expected to specify a local velocity $v_{i}(k)$ on every cell $i$ for every time step $k$. At a given time step of duration $T$, a particle $\mu$ advances according to the local velocity of its current cell. Particle locations within a cell are continuous variables and movement is regarded as continuous in time as well: When $\mu$ crosses a cell boundary during a single move of duration $T$, it can freely choose its next cell (if there is more than one downstream cell) and continue with the velocity encountered there until its available move time ends.

2) Simulation on Variable Time Scales: The simulation scheme on variable time scales can be extended to particle movement. This requires the following completition of the simulation procedure given in Section II-A:

1) Every cell $i$ that is due according to its individual time step length $T_{i}$ calculates supply and demand boundaries from its current occupancy and keeps these results constant for the next $T_{i}$ seconds.

2) All particles $\mu$ that currently reside on a cell $i$ that is due are moved forwards according to the following rules: (i) $\mu$ moves for a duration equal to its start cell $i$ 's time step length $T_{i}$. It might cross several cells during this move if $i$ has a larger $T_{i}$ than its downstream cells. (ii) If $\mu$ has used up its time of movement and arrived on a cell $j$ with $T_{j}>T_{i}$, it continues its move until it has used up an overall movement duration equal to $T_{j}$. This continued move never enters another cell because of Eq. (1) and accounts for the expected waiting time $T_{j}-T_{i}$ until the particle is again due for movement.

3) Every connector $c$ that is due according to its individual time step length $T_{c}$ calculates average flow rates that hold for the next $T_{c}$ seconds and notifies its adjacent cells of the resulting vehicle transmissions.

The workings of step 2 are depicted in Figure 4. Since the particle evaluates all traversed cells' velocities at the start time of its move, the simulation scheme is imprecise only in the order of a time step length, just as the macroscopic simulation logic itself.
Fig. 4. Particle Movement Across Many Cells

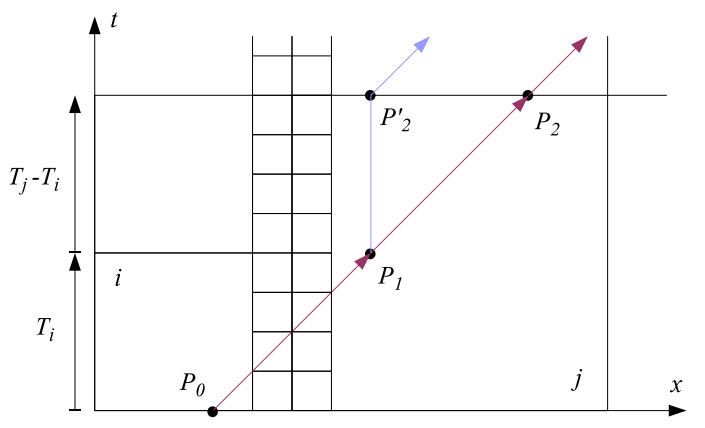

A homogeneous velocity field is assumed so that a correct particle trajectory is depicted by a straight line in the space-time plot. The considered particle starts its move in cell $i$ at space-time point $P_{0}$. During its initial move of duration $T_{i}$ it traverses two small intermediate cells and finally arrives on cell $j$ at point $P_{1}$. If the move was finished there it would not be continued until $T_{j}-T_{i}$ seconds later from point $P_{2}^{\prime}$ because of cell $j$ 's greater time step length $T_{j}$. This would be incorrect as the unstraight trajectory indicates. The particle has to account for the waiting time on cell $j$ by continuing its move for another $T_{j}-T_{i}$ seconds which results in the required linear trajectory through point $P_{2}$.

\section{B. Particle Route Choice}

Having stated the influence of macroscopic dynamics on individual particles, the opposite problem of synchronizing the macroscopic traffic flow with particle behavior is considered next.

1) Specification: The route choice of particle $\mu$ at time step $k$ is expressed by a vector $\mathbf{u}^{\mu}(k)=\left(u_{i j}^{\mu}(k)\right)$ of turning move indicators where $u_{i j}^{\mu}(k)$ is one if $\mu$ proceeds from cell $i$ to $j$ at time step $k$ and zero otherwise. Denoting by $u_{i j}(k)$ the total number of particles that choose turning move $i j$ at time step $k$, the entire population's route choice is expressed by a vector $\mathbf{u}(k)=\left(u_{i j}(k)\right)$ with $\mathbf{u}(k)=\sum_{\mu \in \mathcal{M}} \mathbf{u}^{\mu}(k)$.

An additional state vector $\mathbf{x}^{c n t}(k)=\left(x_{i j}(k)\right)$ is introduced. Each element $x_{i j}(k)$ represents the accumulated number of particles having moved from cell $i$ to $j$ until time step $k$. The dynamics of $\mathbf{x}^{c n t}(k)$ are defined by

$$
\begin{aligned}
\mathbf{x}^{c n t}(0) & =\mathbf{0} \\
\mathbf{x}^{c n t}(k+1) & =\mathbf{x}^{c n t}(k)+\mathbf{u}(k) .
\end{aligned}
$$

From this, macroscopic turning fractions can immediately be obtained through

$$
\beta_{i j}(k)=x_{i j}(k) / \sum_{l} x_{i l}(k) .
$$

These flow splitting fractions can directly be fed into the macroscopic mobility simulation. While update equation (7) assumes time-invariant turning probabilities, a straightforward approach to introduce time variance is to define an additional forgetting parameter $w \in(0,1)$ in a modified turning counter update equation

$$
\mathbf{x}^{c n t}(k+1)=w \mathbf{x}^{c n t}(k)+(1-w) \mathbf{u}(k) .
$$

In the absence of newly observed turning moves this logic causes an exponential forgetting of previously learned 
counts. A useful property of this filter is its infinite memory: If no particles arrive at an intersection for a while, turning counters remain strictly positive and thus ensure well-defined flow splits in Eq. (8).

2) Simulation on Variable Time Scales: If the macroscopic mobility simulation runs on variable time step lengths, particles that cross several connectors during a single move also contribute to all corresponding turning counters. These counters are now updated at variable frequencies themselves:

$$
\begin{aligned}
x_{i j}\left(m T_{c}+n\right) & =x_{i j}\left(m T_{c}\right) m \in \mathbb{N}, n \in\left\{0, \ldots, T_{c}-1\right\} \\
x_{i j}\left(m T_{c}+T_{c}\right) & =w_{c} x_{i j}\left(m T_{c}\right) \\
& +\left(1-w_{c}\right) T_{c}^{-1} \sum_{n=0}^{T_{c}-1} u_{i j}\left(m T_{c}+n\right),
\end{aligned}
$$

where $T_{c}$ is the individual time step duration of the connector $c$ that is crossed by turning move $i j$. An individual weight $w_{c}$ is then necessary for every such connector in order to maintain the same degree of averaging for all turning counters. If input signal $u_{i j}(k)$ is Poissonian with expectation and variance $\lambda$, the variance of $x_{i j}\left(m T_{c}\right)$ approaches

$$
\lim _{m \rightarrow \infty} \operatorname{VAR}\left\{x_{i j}\left(m T_{c}\right)\right\}=T_{c} \lambda \cdot\left(1-w_{c}\right) /\left(1+w_{c}\right) .
$$

The network-wide time constant $\hat{T}$ defined in Section II-B is now employed to postulate that a filtered turning counter's variability must be independent of its connector's time step length and, more specifically, identical to

$$
\operatorname{VAR}\left\{\hat{T}^{-1} \sum_{n=0}^{\hat{T}-1} u_{i j}(m \hat{T}+n)\right\}=\lambda / \hat{T} .
$$

Equating (11) and (12) results in

$$
w_{c}=\left(\hat{T}-T_{c}\right) /\left(\hat{T}+T_{c}\right) .
$$

An infinite memory is still guaranteed if all $T_{c}$ are strictly smaller than $\hat{T}$.

\section{Examples and Model Discussion}

These experiments investigate the proposed mixed micro/macro model's precision as well as the accelerating effect of the simulation scheme on variable time scales. All experiments were conducted on a $1,7 \mathrm{GHz}$ Pentium 4 machine with $1 \mathrm{~GB}$ RAM and implemented in the Java programming language.

A synthetic population of 206'353 travelers with complete daily plans has been generated for the Berlin network introduced in Section II-B [20]. This is a 10 percent sample of Berlin's true motorist population. Since simulations are run on a thinned out version of the full Berlin network, the use of 2 (instead of 10) macroscopic vehicle units per particle already creates realistic congestion patterns. The simulation of a larger microscopic sample is prevented only by the applied machine's limited memory. The following experiments consider the morning rush hour from 6 am to 12 am. More than 16'000 particles are concurrently simulated during the rush hour peak at approximately $9 \mathrm{am}$. Since the
Fig. 5. Precision of Micro/Macro Model Synchronization on a Short Link

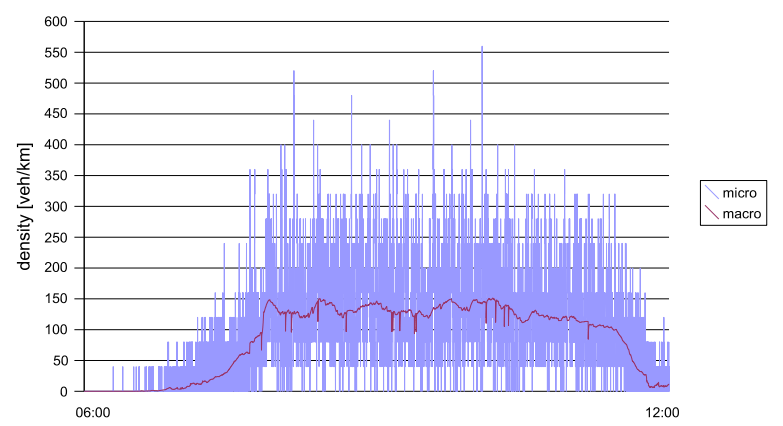

Microscopic and macroscopic density trajectory for a short link of $25 \mathrm{~m}$ length under heavy congestion. The discrete value domain of the microscopic curve reflects the strong vehicle discretization noise. The macroscopic curve removes most of the discretization noise. Unrealistically high microscopic densities are possible because of the massless particles. The macroscopic trend however is within bounds.

Fig. 6. Precision of Micro/Macro Model Synchronization on a Long Link

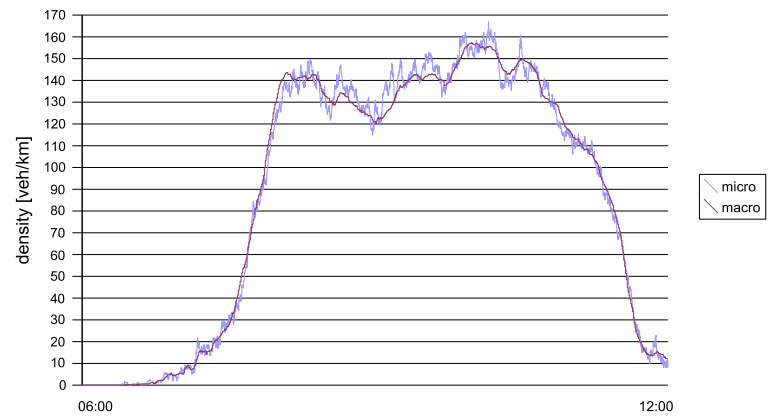

Microscopic and macroscopic density trajectory for a $1.6 \mathrm{~km}$ long link under heavy congestion. Discretization noise has a weaker effect since a greater number of particles is averaged in the microscopic density calculation. The microscopic signal trend is tracked very well by the macroscopic curve.

simulated vehicles' behavior is the outcome of a planning simulation, the network time constant was chosen as large as 5 minutes.

1) Precision of Micro/Macro Coupling: Microscopic behavior influences macroscopic flow splits via the turning counter mechanism while microscopic movement is guided by the macroscopic velocity field. The precision of this micro/macro model synchronization is investigated in the following.

Figures 5 and 6 show microscopic and macroscopic traffic density trajectories for a short link of 25 meters length and for a long link that is 1611 meters long. This difference is reflected in the much greater variance of the microscopic density on the smaller link. Both macroscopic density trajectories track the microscopic trends with high precision and almost no lag. The strong discretization noise on the shorter link is significantly reduced. These results are representative for the network-wide precision of modeling. Further investigations (not shown because of space restrictions) indicate that the coupling scheme preserves the total micro/macro vehicle balance in the network with a negligible error of at most 3 per mille.

The difference between this model and a typical mesoscopic 
Fig. 7. Speedup

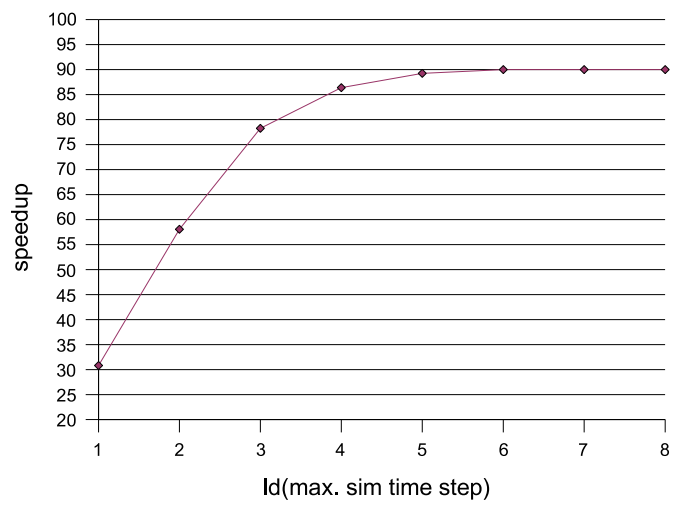

Computational speedup over $\log _{2}$ of largest simulation time step duration in the network.

approach is emphasized: The depicted macroscopic trajectories are not calculated as averages of microscopic vehicle counts on a link. Rather, they implicitly result from continuously tracked turning fractions that guide an appropriate amount of macroscopic flow across that link.

2) Computational Performance: The imprecisions introduced by the simulation scheme on variable time scales can only be justified by its countervailing computational benefits. Figure 7 shows the overall speedup (quotient of simulated time interval and duration of computation) using the same morning peak scenario as before. The maximum speedup is 90 for the considered 2459 link network and an overall population of 206'353 travelers. It has been accomplished one a single-CPU $1.7 \mathrm{GHz}$ Pentium 4 machine. Clearly, this system is ready for real-time simulation of large scenarios.

\section{SUMMARY}

We presented a high performance micro/macro traffic flow simulation. The model owes its ability to simulate large scenarios in multiple real time to the following features: $(i)$ Every link is simulated with an individual time step duration that is optimally adjusted to its characteristics. (ii) The macroscopic mobility simulation only moves non-destination oriented flows. No care has to be taken of partial densities as it would be the case if behavioral aspects were represented macroscopically as well. (iii) The model does not require a realistic number of particles. If, for example, only a 10 percent sample of the complete population is loaded on the network, the macroscopic equivalent of 10 vehicles is inserted into the system together with every particle.

Beyond its computational performance, the following features of the model are noteworthy: (i) The macroscopic model is coupled to the microscopic behavioral model through a filtering mechanism that effectively removes vehicle discretization noise. (ii) Analytical features such as continuity or differentiability of the macroscopic traffic flow model are preserved despite of the microscopic vehicle representation. References [21], [22] describes a first application of this algorithmically beneficial property.

\section{REFERENCES}

[1] M. Lighthill and J. Witham, "On kinematic waves II. A theory of traffic flow on long crowded roads," Proceedings of the Royal Society A, vol. 229, pp. 317-345, 1955.

[2] P. Richards, "Shock waves on highways," Oper. Res., vol. 4, pp. 42-51, 1956.

[3] C. Daganzo, "The cell transmission model: A dynamic representation of highway traffic consistent with the hydrodynamic theory," Transportation Research B, vol. 28B, no. 4, pp. 269-287, 1994.

[4] C. Daganzo, "The cell transmission model, part II: Network traffic," Transportation Research B, vol. 29B, no. 2, pp. 79-93, 1995.

[5] C. Buisson, J. Lebacque, and J. Lesort, "STRADA. A discretized macroscopic model of vehicular traffic flow in complex networks based on the Godunov scheme.", in Symposium on Modelling, Analysis and Simulation, held at CESA 1996 IMACS Multiconference, vol. 2, (Lille, France), pp. 976-981, July 1996.

[6] C. Buisson, J. Lebacque, J. Lesort, and H. Mongeot, "The STRADA model for dynamic assignment," in Proceedings of the 1996 ITS Conference, (Orlando, USA), 1996.

[7] R. LeVeque, Numerical Methods for Conservation Laws. Lectures in Mathematics: ETH Zürich, Basel, Boston, Berlin: Birkhäuser, 1992.

[8] J. Lebacque, "The Godunov scheme and what it means for first order traffic flow models," in Proceedings of the 13th International Symposium on Transportation and Traffic Theory (J.-B. Lesort, ed.), (Lyon, France), Pergamon, July 1996.

[9] S. Lee, "A cell transmission based assignment-simulation model for integrated freeway/ surface street systems." Master Thesis, Ohio State University, 1996.

[10] B. Greenshields, "A study of traffic capacity," in Proceedings Highway Research Board, Washington, DC, vol. 14, pp. 448-477, 1935.

[11] G. Flötteröd and K. Nagel, "Some practical extensions to the cell transmission model," in Proceedings of the 8th IEEE Intelligent Transportation Systems Conference, (Vienna, Austria), IEEE, 2005.

[12] W. Burghout, H. Koutsopoulos, and I. Andreasson, "Hybrid mesoscopic-microscopic traffic simulation," Transportation Research Record, vol. 1934, pp. 218-225, 2005.

[13] S. Espie, D. Gattuso, and F. Galante, "A hybrid traffic model coupling macro and behavioral micro simulation," in Proceedings of the 85. Annual Meeting of the Transportation Research Board, (Washington DC, USA), 2006.

[14] W. Burghout, "Mesoscopic simulation models for short-term prediction," PREDIKT project report CTR2005:03, Centre for Traffic Research (CTR) of Royal Institute of Technology (KTH), Swedish National Road and Transport Research Institute (VTI), and University of Linköping, Stockholm, Sweden, 2005.

[15] E. Cascetta, Transportation systems engineering: theory and methods. Dordrecht: Kluwer Academic Publishers, 2001.

[16] M. Ben-Akiva, M. Bierlaire, D. Burton, H. Koutsopoulos, and R. Mishalani, "Network state estimation and prediction for realtime transportation management applications," Networks and Spatial Economics, vol. 1, pp. 293-318, 2001.

[17] A. De Palma and F. Marchal, "Real cases applications of the fully dynamic METROPOLIS tool-box: And advocacy for large-scale mesoscopic transportation systems," Networks and Spatial Economics, vol. 2, pp. 347-369, 2002.

[18] H. S. Mahmassani, "Dynamic network traffic assignment and simulation methodology for advanced system management applications," Networks and Spatial Economics, vol. 1, no. 3/4, pp. 267-292, 2001.

[19] K. Nökel and M. Schmidt, "Parallel DYNEMO: Meso-scopic traffic flow simulation on large networks," Networks and Spatial Economics, vol. 2, pp. 387-403, December 2002.

[20] M. Rieser, K. Nagel, U. Beuck, M. Balmer, and J. Rümenapp, "Truly agent-oriented coupling of an activity-based demand generation with a multi-agent traffic simulation," in Proceedings of the 86. Annual Meeting of the Transportation Research Board, (Washington DC, USA), 2007.

[21] G. Flötteröd and K. Nagel, "Modeling and estimation of combined route and activity location choice," in Proceedings of the 9th IEEE Intelligent Transportation Systems Conference, (Toronto, Canada), IEEE, 2006.

[22] G. Flötteröd and K. Nagel, "State estimation for multi-agent simulations of traffic," in Proceedings of the 2007 World Conference on Transport Research, (Berkley, California), June 2007. 